\title{
Desenvolvimento pós-embrionário de Cochliomyia macellaria (Fabricius) (Diptera: Calliphoridae), criada em duas dietas naturais, sob condições controladas
}

\author{
Débora Cardoso da Silva ${ }^{1,3 *}$ \\ Valéria Magalhães Aguiar Coelho ${ }^{2}$ \\ Sandra Lúcia da Cunha e Silva ${ }^{3}$ \\ Rafaela Pereira de Carvalho ${ }^{2}$ \\ Gonzalo Efrain Moya Borja ${ }^{1}$

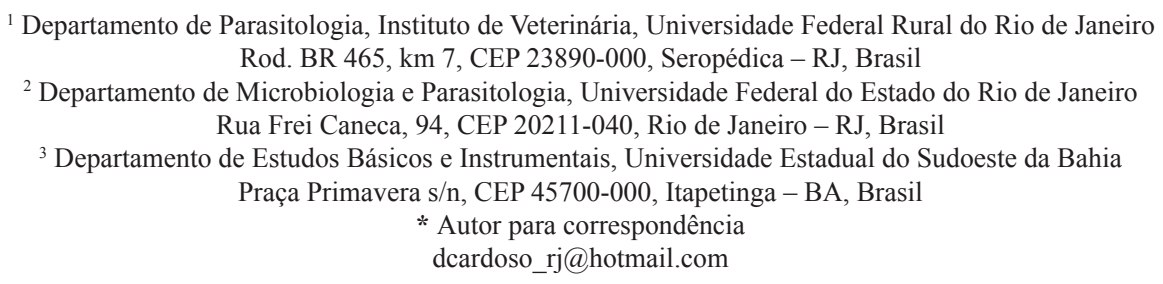

Submetido em 04/05/2012

Aceito para publicação em 31/08/2012

\section{Resumo}

Cochliomyia macellaria tem interese médico e médico veterinário por ser veiculadora de enteropatógenos e causadora de miíase secundária. A busca por dietas com baixo custo que mantenham a qualidade da criação torna-se necessária. Objetivou-se avaliar a dieta moela de frango no desenvolvimento pós-embrionário de $C$. macellaria, comparada a carne bovina. Quarenta neolarvas ( $3^{\mathrm{a}}$ geração) foram transferidas para $80 \mathrm{~g}$ de dieta (quatro repetições/tratamento). Foi registrada a massa corporal das larvas maduras, e estas transferidas para tubos de ensaio e mantidas a $\mathrm{T} 30^{\circ} \mathrm{C}$ dia $/ 28^{\circ} \mathrm{C}$ noite, UR $70 \pm 10 \%$ e $12 \mathrm{~h}$ de fotofase. As observações foram diárias. Houve diferença significativa entre a massa corporal das larvas criadas na dieta moela de frango $(0,067 \mathrm{~g})$ e carne bovina $(0,062 \mathrm{~g})$. Não houve diferença significativa entre a duração média em dias do estágio larval (4,00; $4,17)$, pupal $(4,09 ; 4,04)$; de neolarva a adulto $(8,07$ e 8,16$)$, para moela e carne, respectivamente. A viabilidade média larval, pupal e total (> 85\%) não diferiram estatisticamente pelo teste t de Student, 5\% de significância. Não houve desvio da razão sexual e não foi observada anormalidade dos adultos. A dieta moela de frango é uma alternativa eficaz e de baixo custo para criação de C. macellaria em laboratório.

Palavras-chave: Biologia de laboratório; Criação de imaturos; Miíase; Mosca varejeira

\section{Abstract}

Post-embryonic development of Cochliomyia macellaria (Fabricius) (Diptera: Calliphoridae), reared on two natural diets, under controlled conditions. Cochliomyia macellaria is of medical and veterinary interest, because it is an enteropathogen vector and causes secondary miyasis, and low cost diets are needed 
that maintain rearing quality of this species. The objective of this study was to assess a chicken gizzard diet, compared to beef, in the post-embrionic development of C. macellaria. Forty neolarvas (3rd generation) were transferred to $80 \mathrm{~g}$ diets (four replications/treatment). The body mass of the mature larvae was recorded and the larvae were transferred to test tubes, kept at $\mathrm{T} 30^{\circ} \mathrm{C}$ day $/ 28^{\circ} \mathrm{C}$ night, $\mathrm{RH} 70 \pm 10 \%, 12$ hours light period, and observed daily. There was a significant difference between the body mass of larvae reared on the chicken gizzard $(0.067 \mathrm{~g})$ and beef $(0.062 \mathrm{~g})$ diets. There was no significant difference between the mean duration in days of the larval $(4.00 ; 4.17)$, pupa $(4.09 ; 4.04)$ and neolarva to adult $(8.07$ and 8.16$)$ stages based on the gizzard and beef diets, respectively. The mean larval, pupa and total viability $(>85 \%)$ did not differ statistically by the Student's t test (at 5\% significance). There was no deviation in the sex ratio and no abnormality was observed in the adults. The results suggest that the chicken gizzard diet is an efficacious and cheap alternative for rearing C. macellaria in the laboratory.

Key words: Blow fly; Juvenile rearing; Laboratory biology; Miyasis

\section{Introdução}

Cochliomyia macellaria (Fabricius, 1775) é encontrada em regiões tropicais e subtropicais do Novo Mundo, desde o Sul do Canadá até Argentina (FERREIRA, 1983; BAUMGARTNER; GREENBERG, 1985). É uma das principais espécies causadora de miĺase secundária, contribuindo assim para agravar um quadro infeccioso já estabelecido (GOMES et al., 2000) sendo também reconhecida como veiculadora de ovos de Dermatobia hominis (Linnaeus Jr., 1781) responsável pela miíase furuncular cutânea (GUIMARÃES; PAPAVERO, 1999; MOYA BORJA, 2003). Os adultos de C. macellaria são atraídos por vários substratos para alimentação ou postura como resíduos de origem humana e animal, carcaças, entre outros. Neste sentido esta espécie tem sido implicada como veiculadora mecânica de enteropatógenos em humanos (THYSSEN et al., 2004; GRACZYK et al., 2005; RIBEIRO et al., 2011) e em animais (GREENBERG, 1971), bem como tem sido registrada em estudos de sucessão ecológica em carcaças (OLIVEIRA-COSTA; MELLO-PATIU, 2004; BATTAN et al., 2007; SHARANOWSKI et al., 2008; GOMES et al., 2009; OLIVEIRA; VASCONCELO, 2010).

Diante da importância de realização de estudos com essa espécie, seja para a medicina humana ou animal, faz-se necessário o estabelecimento e criação de colônia desses califorídeos em laboratório, para que se possam traçar estratégias que auxiliem em diferentes estudos no campo da entomologia pura e aplicada.

No que diz respeito ao hábito alimentar quando mantidas em laboratório, a dieta natural utilizada para criação de larvas de califorídeos é a carne bovina e equina (MARCKENKO, 1985; QUEIROZ; MILWARDDE-ZEVEDO, 1991; CUNHA-E-SILVA; MILWARDDE-AZEVEDO, 1994). Contudo, alguns fatores como o alto custo e acessibilidade do produto, têm levado à busca por substratos alternativos que possam substituí-las, sem perder de vista a qualidade da colônia (BARBOSA et al., 2004; SOUZA et al., 2010; FERRAZ et al., 2011; 2012). Vale ressaltar que a nutrição larval exerce uma forte influência sobre o tamanho dos adultos, existindo uma correlação direta entre o tamanho do adulto e a taxa de oviposição (THOMAS, 1993).

Dentro desse contexto a moela de frango é potencialmente promissora, visto ser um produto acessível e ter custo inferior a dieta tradicionalmente utilizada. Além disso, o conteúdo nutricional em moela de frango é semelhante à carne bovina (ESPOSITO et al., 2009). Neste sentido o presente trabalho objetivou avaliar o desenvolvimento pós-embrionário de $C$. macellaria criadas em duas dietas naturais, a moela de frango e a carne bovina.

\section{Material e Métodos}

A criação estoque dos muscóides e a parte experimental foram realizadas no Laboratório de Estudos de Dípteros, do Departamento Microbiologia e Parasitologia da Universidade Federal do Estado do Rio de Janeiro (UNIRIO). A criação foi estabelecida a partir de adultos coletados no Campus da Universidade Federal Rural do Rio de Janeiro, município de Seropédica - RJ, latitude $22^{\circ} 45^{\prime} 48^{\prime \prime}$ S e longitude $43^{\circ} 41^{\prime} 23^{\prime \prime} \mathrm{W}$. Para a 
coleta dos adultos foi utilizada armadilha seguindo o modelo de Mello et al. (2007), a isca utilizada foi peixe fresco (sardinha) e a armadilha foi exposta por cerca de 6 h durante o dia. A identificação foi realizada com auxílio de chave taxonômica (MELLO, 2003). A metodologia de criação seguiu a orientação preconizada por Cunha-e-Silva e Milward-de-Azevedo (1996) e Barbosa et al. (2008).

$\mathrm{Na}$ etapa experimental a carne bovina (patinho) e a moela de frango foram mantidas por $72 \mathrm{~h}$ a uma temperatura de $12^{\circ} \mathrm{C}$, e transferidas à temperatura ambiente por $24 \mathrm{~h}$ antes do início do experimento. A carne foi cortada em pedaços de aproximadamente $2 \mathrm{~cm}^{2}$ e a moela foi cortada ao meio. As fêmeas foram estimuladas a ovipositar em carne bovina. Logo após a eclosão, 40 neolarvas da $3^{\text {a }}$ geração foram transferidas com auxílio de um pincel $\mathrm{n}^{\circ} 01$ para Beckers $(100 \mathrm{ml})$ contendo $80 \mathrm{~g}$ da dieta. Estes recipientes foram colocados dentro de Beckers maiores $(400 \mathrm{ml})$ contendo maravalha esterilizada, para servir de substrato após o abandono espontâneo da dieta, e então foram fechados com tecido de náilon e presos com elástico. Foram utilizados quatro repetições por tratamento. Logo após o abandono da dieta, foram formados lotes de cinco larvas e registrados a massa corporal em balança analítica, transferidas para tubos de ensaio contendo maravalha esterilizada que serviu de substrato de pupariação, vedadas com tecido de náilon e presos com elástico. Após a emergência, os adultos foram sexados e observados quanto à sua anormalidade morfológica. $\mathrm{O}$ estudo foi conduzido em câmara climatizada regulada a temperatura de $30^{\circ} \mathrm{C}$ dia $/ 28^{\circ} \mathrm{C}$ noite, $70 \pm 10 \%$ UR e $12 \mathrm{~h}$ de fotofase.

As observações foram diárias sempre no mesmo horário, no período matutino, até a emergência dos adultos. O delineamento experimental foi inteiramente casualizado. A comparação entre as médias da massa corporal das larvas, a duração e a viabilidade dos estágios larval, pupal, e total (neolarva a adulto), bem como a normalidade dos adultos foram analisadas pelo Teste $\mathrm{t}$ de Student $(\alpha=5 \%)$. A razão sexual foi testada em relação à frequência esperada, pelo teste do quiquadrado $\left(\chi^{2}\right)$.

\section{Resultados e Discussão}

Observou-se que a média da massa corporal das larvas maduras criadas na dieta a base de moela de frango, $0,067 \mathrm{~g}$, foi significativamente maior $(\mathrm{P}=0,0338)$ quando comparado com as larvas criadas na dieta a base de carne bovina, cujo peso médio foi de $0,062 \mathrm{~g}$ (Tabela 1).

Cunha-e-Silva e Milward-de-Azevedo (1994) obtiveram peso médio de $0,056 \mathrm{~g}$, em larvas de $C$. macellaria, quando criadas em carne equina, peso inferior ao presente trabalho. Fato que pode ser atribuído à quantidade de dieta oferecida aos insetos. Estes autores utilizaram a relação de cerca de $1 \mathrm{~g}$ de alimento para 1,5 larvas, enquanto que no presente trabalho a relação foi de $2 \mathrm{~g}$ de dieta para uma larva. Aguiar-Coelho e Milwardde-Azevedo (1996) ao realizarem experimentos de densidade larval de C. macellaria, em dieta que consistia de carne equina, demonstraram que a relação de $1 \mathrm{~g}$ de carne para uma larva, é ideal para criação desta espécie. Cunha-e-Silva e Milward-de-Azevedo (1999) utilizaram a relação $4 \mathrm{~g}$ de alimento, carne equina, para uma larva de $C$. macellaria e observaram pesos que oscilaram entre 0,06 a $0,07 \mathrm{~g}$. A quantidade de dieta utilizada no presente estudo foi considerada satisfatória, pois as

TABELA 1: Massa corporal de larvas maduras e duração do desenvolvimento pós-embrionário (dias) de Cochliomyia macellaria, criadas em dieta moela de frango e carne bovina em condições controladas $\left(30^{\circ} \mathrm{C}\right.$ dia/ $28^{\circ} \mathrm{C}$ noite, $70 \pm 10 \%$ UR e $12 \mathrm{~h}$ de fotofase).

\begin{tabular}{ccccc} 
Dieta & $\begin{array}{c}\text { Massa corporal de larvas } \\
(\mathbf{g})\end{array}$ & $\begin{array}{c}\text { Estágio } \\
\text { larval }\end{array}$ & $\begin{array}{c}\text { Estágio } \\
\text { pupa }\end{array}$ & $\begin{array}{c}\text { Período de Neolarva à } \\
\text { emergência }\end{array}$ \\
\hline & $\mathrm{X} \pm \mathrm{dp}$ & $\mathrm{X} \pm \mathrm{dp}$ & $\mathrm{X} \pm \mathrm{dp}$ & $\mathrm{X} \pm \mathrm{dp}$ \\
Moela & $0,067 \pm 0,001 \mathrm{a}$ & $4,00 \pm 0,000 \mathrm{a}$ & $4,09 \pm 0,055 \mathrm{a}$ & $8,07 \pm 0,058 \mathrm{a}$ \\
Carne & $0,062 \pm 0,002 \mathrm{~b}$ & $4,17 \pm 0,128 \mathrm{a}$ & $4,04 \pm 0,096 \mathrm{a}$ & $8,16 \pm 0,054 \mathrm{a}$ \\
\hline
\end{tabular}

$\mathrm{X}=$ Média, dp = Desvio padrão; Valores seguidos pela mesma letra na mesma coluna não diferem significativamente pelo Teste $\mathrm{t}$, ao nível de $5 \%$. 
larvas atingiram massa corporal próxima ou superior a estudos anteriores realizados com esta espécie.

Nesse estudo foram utilizadas duas dietas naturais, que segundo definição de Panizzi e Parra (2009), tratase de uma dieta obtida da natureza, podendo apresentar qualidade nutricional variável. Essa variação nutricional pode ter contribuído com a diferença no peso larval observado. A moela de frango possui em sua composição média $0,56 \%$ de lipídios e $20,19 \%$ de proteína (PEREIRA et al., 2002), enquanto a carne bovina (patinho) possui 4,2\% de lipídios e $21,7 \%$ de proteína (NEPA, 2011), demonstrando, aparentemente, uma maior disponibilidade de lipídios e proteínas. Contudo, deve-se ressaltar o índice de eficiência alimentar, como um fator que pode ter influenciado no peso das larvas. Embora a carne bovina apresente um maior percentual de lipídios, pode ter ocorrido uma maior eficiência na conversão alimentar pelas larvas criadas na moela de frango. Norris (1965) e Campobasso et al. (2001) citam que as larvas sapronecrófagas podem apresentar diferentes taxas de desenvolvimento quando invadem diferentes tipos de tecidos, em diferentes órgãos, podendo estar relacionado ao valor nutricional ou à disponibilidade dos nutrientes. Vale destacar que após o abandono das larvas maduras das dietas observou-se, no recipiente de criação (Becker), uma maior quantidade de resíduos sólidos (dieta) no tratamento carne bovina, enquanto no tratamento moela de frango o resíduo final foi quase inexistente e de consistência liquefeita. Provavelmente as larvas consumiram uma maior quantidade de dieta moela, em um mesmo intervalo de tempo, o que pode ter contribuído para um aumento da massa corporal. A consistência dessa dieta associada à ação das larvas, pode ter possibilitado uma decomposição mais acelerada, quando comparada à carne, tornando o meio (moela) mais liquefeito.

Por serem as dietas utilizadas nesse estudo um meio xênico, estudos posteriores devem ser realizados no sentido de se avaliar a composição microbiana das diferentes dietas e se estas interagem, de alguma forma, com C. macellaria, acelerando o processo de decomposição ou até mesmo promovendo, a partir dessa interação, uma maior disponibilização de nutrientes.

Nos demais parâmetros avaliados não houve diferença estatística significativa. O ritmo de abandono das larvas, espontaneamente em ambas as dietas, foi de três dias. A média da duração do estágio larval foi de 4,0 dias (Tabela 1). Greenberg e Szyska (1984), ao utilizarem uma dieta que também apresentava um elevado valor protéico (peixe) em diferentes condições ambientais (temperatura mínima de 26,0 e máxima de $27,1^{\circ} \mathrm{C}$ ) reportaram um período larval semelhante ao presente estudo, aproximadamente 4,0 dias. Cunha-e-Silva e Milward-de-Azevedo (1999) observaram um período médio inferior, de 3,3 dias em larvas criadas em carne equina, sob a temperatura de $30^{\circ} \mathrm{C}$.

Embora não tenha havido diferença significativa na duração do desenvolvimento do estágio larval (Tabela 1), ao analisar o ritmo de pupariação, $100 \%$ das larvas criadas na moela de frango pupariaram no 4을 dia após o início do experimento, enquanto que as criadas em carne bovina o pico de pupariação foi no $4^{\circ}$ dia (89\%), com percentuais menores no $5^{\text {a }}(9 \%)$ e $6^{\circ}$ dia $(2 \%)$. Estes resultados revelam uma maior uniformidade de comportamento dos insetos criados na dieta moela de frango. Cunha-e-Silva e Milward-de-Azevedo (1994), registraram pupariação em $C$. macellaria alimentadas

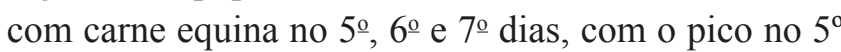
dia, demonstrando padrão semelhante ao da carne bovina no presente estudo com mais de $70 \%$ dos indivíduos pupariando no mesmo dia.

Não houve diferença significativa no estágio pupal nas duas dietas estudadas, apresentando duração média de 4,0 dias (Tabela 1), resultado semelhante foi obtido em outros estudos como o de Paes e Milward-deAzevedo (1998) e Cunha-e-Silva e Milward-de-Azevedo (1999), utilizando como dietas peixe e carne equina, respectivamente.

No presente estudo observou-se uma viabilidade larval de $100 \%$ para carne bovina, enquanto que para moela foi de $95,50 \%$. Não houve diferença significativa na viabilidade pupal nas dietas moela de frango e carne; $89,12 \%$ e $87,50 \%$, respectivamente. Porém, a viabilidade total foi numericamente superior para os imaturos criados em dieta moela $85 \%$ comparado com os criados em carne 79,25\% (Tabela 2), embora não apresente diferença significativa. Tais resultados demonstram que a dieta moela de frango é promissora para criação de larvas desta espécie, corroborando com estudos que consideram 
TABELA 2: Viabilidade média do estágio larval, pupal e total (neolarva a emergência do adulto), razão sexual e taxa de adultos normais de Cochliomyia macellaria, criadas em dieta de moela de frango e carne bovina, em condições controladas $\left(30^{\circ} \mathrm{C} \mathrm{dia} / 28^{\circ} \mathrm{C}\right.$ noite, $70 \pm 10 \%$ UR e 12 horas de fotofase).

\begin{tabular}{ccccccc}
\multirow{2}{*}{ Dieta } & \multicolumn{3}{c}{ Viabilidade (\%) } & \multirow{2}{*}{ Razão Sexual } & Taxa de Normalidade \\
\cline { 2 - 4 } & Larval & Pupal & Total & & 100 \\
Moela & $95,50 \mathrm{a}$ & $89,12 \mathrm{a}$ & $85,00 \mathrm{a}$ & 0,49 & 100 \\
Carne & $100 \mathrm{a}$ & $87,50 \mathrm{a}$ & $79,25 \mathrm{a}$ & 0,48 & \\
\hline
\end{tabular}

Valores seguidos pela mesma letra na mesma coluna não diferem significativamente pelo Teste t, ao nível de 5\%.

Razão sexual = № fêmeas/ № machos+№ fêmeas.

uma dieta eficiente a que apresenta uma viabilidade larval acima de $60 \%$ (LOUREIRO et al., 2005).

Com relação ao período de desenvolvimento de C. macellaria da transferência de neolarvas até a emergência dos adultos não houve diferença significativa entre as duas dietas avaliadas, observou-se valores médios de 8,07 dias e 8,16 dias para os insetos criados em moela de frango e carne bovina, respectivamente (Tabela 1). Estes dados não divergem dos resultados obtidos por Cunha-e-Silva e Milward-de-Azevedo (1999) que trabalharam com a dieta carne equina $\left(27^{\circ} \mathrm{C}-30^{\circ} \mathrm{C}\right.$ de temperatura), enquanto que Greenberg e Szyska (1984) observaram uma extensão deste período, cerca de dez dias, fato justificado pela baixa temperatura a que os insetos foram submetidos neste trabalho.

Para ambas as dietas, a razão sexual não divergiu do esperado cerca de $50 \%$, para a dieta carne, $50,9 \%$ machos e $49,1 \%$ fêmeas, enquanto que para a dieta moela $48,03 \%$ machos e $52 \%$ fêmeas, demonstrando que as proporções observadas estão de acordo com as esperadas (Tabela 2). Todos os tratamentos apresentaram $100 \%$ de adultos normais (Tabela 2).

Ao avaliarem o desenvolvimento de imaturos de Chrysomya putoria (Wiedemann, 1818), criadas em dieta natural moela, Ferraz et al. (2012) não observaram diferença significativa na massa corporal, duração do estágio larval e estágio pupal, na viabilidade de larvas e pupas não observaram desvio da razão sexual e anormalidade dos adultos quando comparados a dieta carne bovina, demonstrando a mesma tendência do presente trabalho, que a dieta moela é uma dieta promissora para a referida espécie.

Uma alimentação de qualidade constitui a condição básica para o crescimento, o desenvolvimento e a reprodução dos insetos. A quantidade e a qualidade do alimento consumido na fase larval interferem no crescimento, no tempo do desenvolvimento, no peso do corpo, na sobrevivência, bem como influenciam a fecundidade, a longevidade, a movimentação e a capacidade de competição dos adultos (PANIZZI et al., 2009). É fundamental destacar, também, que o alimento natural apresenta variação na qualidade e, não raro, ocorre a presença de aleloquímicos ou produtos do metabolismo secundário que podem ser tóxicos. Além disso, a localização anatômica do tecido muscular pode interferir na mobilidade dos muscóides que utilizam suas carcaças como fonte alimentar.

Pelos resultados observados, a moela de frango é uma potencial dieta natural para criação de larvas de C. macellaria, podendo substituir a carne bovina e equina. Além disso, a moela mostra-se mais vantajosa por ter preço mais baixo que a carne bovina, ser de fácil aquisição no mercado, e seu manuseio ser mais simples que a carne bovina. Contudo, devem-se realizar estudos no sentido de se avaliar a interferência da dieta moela de frango no potencial reprodutivo de C. macellaria, visto que o substrato proteico é importante para o amadurecimento dos ovócitos (WALL et al., 2002), para o estímulo da cópula e oviposição (BARTON-BROWNE et al., 1976) em califorídeos necrobiontófagos.

\section{Referências}

AGUIAR-COELHO, V. M.; MILWARD-DE-AZEVEDO, E. M. V. Relações intra-específicas de Cochliomyia macellaria (Fabr), Chrysomya albiceps (Wied) e Chrysomya megacephala (Fab) (Dipter, Calliphoridae) em condições experimentais. Revista Brasileira de Entomologia, São Paulo, v. 40, n. 1, p. 35-40, 1996. BATTAN HORENSTEIN, M.; LINHARES, A. X.; ROSSO, B.; GARCIA, M. D. Species composition and seasonal succession of 
saprophagous calliphorids in a rural area of Cordoba, Argentina. Biologycal Research, Santiago, v. 40, p. 163-171, 2007.

BARBOSA, L. S.; COURI, M. S.; COELHO, V. M. A. Influência do aumento do número de pupas hospedeiras de Cochliomyia macellaria (Diptera, Calliphoridae) no desenvolvimento do parasitóide Nasonia vitripennis (Hymenoptera, Pteromalidae) em laboratório. Iheringia, Série Zoologia, Porto Alegre, v. 98, n. 3, p. 339-344, 2008.

BARBOSA, L. S.; JESUS, D. M. L.; COELHO, V. M. A. Longevidade e capacidade reprodutiva de casais agrupados de Chrysomya megacephala (Fabricius, 1794) (Diptera, Calliphoridae) oriundos de lavras criadas em dieta natural e oligídica. Revista Brasileira de Zoociências, Juiz de Fora, v. 6, n. 2, p. 207-217, 2004.

BARTON-BROWNE, L.; BARTEL, R. J.; VAN GERWEN, A. C. M.; LAERENCE, L. A. Relationship between protein ingestion and sexual receptivity in females of the Australian sheep blowfly Lucilia cuprina. Physiological Entomology, London, v. 1, n. 4, p. 235-240, 1976.

BAUMGARTNER, D. L.; GREENBERG, B. Distribution and Medical Ecology of the Blow Flies (Diptera: Calliphoridae) of Peru. Annals of the Entomological Society of America, Lanham, v. 78, n. 5, p. 565-587, 1985.

CAMPOBASSO, P. C.; DI VELLA, G.; INTRONA, F. Factors affecting decomposition and Diptera colonization. Forensic Science International, Lausanne, v. 120, n. 1-2, p. 18-27, 2001.

CUNHA-E-SILVA, S. L.; MILWARD-DE-AZEVEDO, E. M. V. Estudo comparado do desenvolvimento pós-embrionário de Cochliomyia macellaria (Fabricius) (Diptera, Calliphoridae) à base de carne em laboratório. Revista Brasileira de Zoologia, Curitiba, v. 11, n. 4, p. 659-668, 1994.

CUNHA-E-SILVA, S. L.; MILWARD-DE-AZEVEDO, E. M. V. Aspectos da biologia da reprodução e longevidade de Cochliomyia macellaria (Fabricius) (Diptera, Calliphoridae), em condições experimentais. I. Casais agrupados. Revista Brasileira de Zoologia, Curitiba, v. 13, n. 4, p. 883-889, 1996.

CUNHA-E-SILVA, S. L.; MILWARD DE AZEVEDO, E. M. V. Controle de qualidade de imaturos de Cochliomyia macellaria (Fabricius) (Diptera: Calliphoridae) em estoque. Parasitologia al Día, Santiago, v. 23, n. 1-2, 1999.

ESPOSITO, A. B. M.; LIMA, C. C.; SOUZA, F. N.; RIBAS, F.; KOROLHUK, J; LUZ, K. C.; MUNARO, V.; RIBAS, A. R.; BALBI, M. E. Avaliação de miúdos de Gallus domesticus como fonte proteica.Visão Acadêmica, Curitiba, v. 10, n. 2, p. 59-74, 2009.

FERRAZ, A. C. P.; BOSISIO, D. D.; AGUIAR-COELHO, V. M. Dieta para larvas de Chrysomya megacephala, Chrysomya putoria $\mathrm{e}$ Cochliomyia macellaria (Diptera: Calliphoridae). EntomoBrasilis, Vassouras, v. 42, n. 3, p. 125-129, 2011.

FERRAZ, A. C. P.; DALlaVECCHIA D. L.; SILVA, D. C.; CARVALHO, R. P.; SILVA FILHO, R. G.; AGUIAR-COELHO, V. M. Alternative diets for Chrysomya putoria, an Old World screwworm fly. Journal of Insect Science, Tucson, v. 12, n. 43, p. 1-11, 2012.

FERREIRA, M. J. M. Sinantropia de Calliphoridae (Diptera) em Goiania, Goiás. Revista Brasileira de Biologia, Rio de Janeiro, v. 43, n. 2, p. 199-210, 1983.
GOMES, A.; KOLLER, W. W.; BARROS, T. M. Sazonalidade da mosca-varejeira Cochliomyia macellaria (Diptera, Calliphoridae), na região do Cerrado, Campo Grande, MS. Revista Brasileira de Parasitologia Veterinária, São Carlos, v. 9, n. 2, p. 125-128, 2000. GOMES, L.; GOMES, G.; DESUÓ, I. C. A preliminary study of insect fauna on pig carcasses located in sugarcane in winter in southeastern Brazil. Medical and Veterinary Entomology, Oxford, v. 23, n. 2, p. 155-159, 2009.

GRACZYK, T.; KNIGHT, R.; TAMANG, L. Mechanical transmission of human protozoan parasites by insects. Clinical Microbiology Reviews, Washington, v. 1, n. 18, p. 128-132, 2005. GREENBERG, B. Flies and Disease: Ecology, classification and biotic associations. Vol. I. New York: Princeton University Press, 1971. 865 p.

GREENBERG, B.; SZYSKA, M. L. Immature stages and biology of 15 species of Peruvian Calliphoridae, Diptera. The Entomological Society of America, Lanham, v. 77, p. 488-517, 1984.

GUIMARÃES, J. H.; PAPAVERO, N. Myiasis in man and animals in the Neotropical Region: bibliographic database. São Paulo: Editora Plêiade/Fapesp, 1999. 308 p.

LOUREIRO, M. S.; OLIVEIRA, V. C.; D’ALMEIDA, J. M. Desenvolvimento pós-embrionário de Pattonella intermutans (Thomson) (Diptera: Sarcophagidae) em diferentes dietas. Revista Brasileira de Entomologia, São Paulo, v. 49, n. 1, p. 127-129, 2005.

MARKENCO, M. I. Development of Chrysomya albiceps (Wiedman) (Diptera: Calliphoridae). Entomologicheskoe Obozrenie, Petersburg, v. 1, p. 79-84, 1985.

MELLO, R. P. Chave para identificação das forma adultas da espécies da família Calliphoridae (Diptera: Brachycera, Cyclorrhapha) encontradas no Brasil. Revista Entomología y Vectores, Rio de Janeiro, v. 10, n. 2, p. 255-268, 2003.

MELlO, R. S.; QUEIROZ, M. M. C.; AGUIAR-COELHO, V. M. Population fluctuations of calliphorid species (Diptera, Calliphoridae) in the Biological Reserve of Tinguá, state of Rio de Janeiro, Brazil. Iheringia Série Zoologia, Porto Alegre, v. 97, n. 4, p. 48-485, 2007.

MOYA-BORJA, G. E. 2003. Erradicação ou manejo integrado das miíases neotropicais das Américas. Pesquisa Veterinária Brasileira, Rio de Janeiro, v. 23, p. 131-138, 2003.

NEPA. Tabela brasileira de composição de alimentos. 4 ed. Campinas: NEPA/UNICAMP, 2011. 161 p.

NORRIS, K. R. The bionomics of blowflies. Annual Review of Entomology, Palo Alto, v. 10, p. 47-68, 1965.

OLIVEIRA, T. C.; VASCONCELO, S. D. Insects (Diptera) associated with cadavers at the Institute of Legal Medicine in Pernambuco, Brazil: Implications for forensic entomology. Forensic Science International, Lausanne, v.198, n. 1-4, p. $97-$ $102,2010$.

OLIVEIRA-COSTA, J.; MELLO-PATIU, C. A. Application of Forensic Entomology to estimate of the postmortem interval (PMI) in homicide investigations by the Rio de Janeiro Police Department in Brazil. Journal of Forensic Medicine and Toxicology, New Delhi, v. 5, n. 1, p. 40-44, 2004.

PAES, M. J.; MILWARD-DE-AZEVEDO, E. Desenvolvimento pósembrionário de Cochliomyia macellaria (Fabricius) (Diptera: 
Calliphoridae) criada em dietas naturais processadas em condições controladas. Parasitologia al Día, Santiago, n. 22, p. 3-4, 1998.

PANIZZI, A. R.; PARRA, J. R. P. Bioecologia e nutrição de insetos: base para o manejo integrado de pragas. 1 ed. Brasília: EMBRAPA/ CNPq, 2009. 1164 p.

PEREIRA, N. R.; MUNIZ, E. C.; MATSUSHITA, M.; SOUZA, N. E. Cholesterol and fatty acids profile of brazilian commercial chicken giblets. Archivos Latinoamericanos de Nutrición, Caracas, v. 52, n. 2, p. 203-206, 2002.

QUEIROZ, M. M. C.; MILWARD-DE-AZEVEDO, E. M. V. Técnicas de criação e alguns aspectos da biologia de Chrysomya albiceps (Wiedemann) (Diptera, Calliphoridae), em condições de laboratório. Revista Brasileira de Zoologia, Curitiba, v. 8, p.7584, 1991.

RIBEIRO, M. J. R; DIAS, M. F. D.; TESHIMA, E.; BARBONI, A. R. Insalubridade ambiental e aspectos sociais associados a patógenos intestinais isolados de dípteros. Revista Engenharia Sanitária e Ambiental, Rio de Janeiro, v. 6, n. 1, p. 83-90, 2011.
SHARANOWSKI, B. J.; WALKER, G. E.; ANDERSON, G. S. Insect succession and decomposition patterns on shaded and sunlit carrion in Saskatchewan in three different seasons. Forensic Science International, Lausanne, v. 79, n. 2-3, p. 219-40, 2008.

SOUSA, A. G. P.; FERRAZ, A. C. P.; NASCIMENTO, A. L. O.; AGUIAR-COELHO, V. M. Alternative natural diet for the creation of immature oriental latrine flies under controlled conditions. Revista Brasileira de Zoociências, Juiz de Fora, v. 12, n. 2, p. 133-140, 2010

THOMAS, D. B. Fecundity and oviposition in laboratory colonies of the screwworm fly (Diptera, Calliphoridae). Jounal of Economic Entomology, Lanham, v. 86, n. 5, p. 1464-1472, 1993.

THYSSEN, P. J.; MORETTI, T. C.; UETA M. T.; RIBEIRO, O. B. O papel de insetos (Blattodea, Diptera e Hymenoptera) como possíveis vetores mecânicos de helmintos em ambiente domiciliar e peridomiciliar. Cadernos de Saúde Pública, Rio de Janeiro, v. 20, n. 4, p. 1096-1102, 2004.

WALL, R.; WEARMOUTH, V. J.; SMITH, K. E. Reproductive allocation by the blow fly Lucilia sericata in response to protein limitation. Physiological Entomology, London, v. 27, n. 4, p. $267-$ 274, 2002. 